\title{
Failure of remission induction by glucocorticoids alone or in combination with immunosuppressive agents in IgG4-related disease: a prospective study of 215 patients
}

Liwen Wang ${ }^{1,2 \dagger}$, Panpan Zhang ${ }^{1 \dagger}$, Mu Wang ${ }^{3}$, Ruie Feng ${ }^{4}$, Yamin Lai ${ }^{5}$, Linyi Peng ${ }^{1}$, Yunyun Fei ${ }^{1}$, Xuan Zhang ${ }^{1}$, Yan Zhao ${ }^{1}$, Xiaofeng Zeng ${ }^{1}$, Fengchun Zhang ${ }^{1}$ and Wen Zhang ${ }^{*^{*}}$ (D)

\begin{abstract}
Background: The aim of this study was to assess the outcomes of remission induction in patients with lgG4-related disease (IgG4-RD) in our cohort, and to investigate the characteristics, prognosis, and risk factors in the patients failed of remission induction.

Methods: We prospectively enrolled 215 newly diagnosed patients with lgG4-RD, who were initially treated with glucocorticoid (GC) alone or in combination with immunosuppressive agents (IM), and had at least 6 months of follow up. The therapeutic goals of remission induction were defined as fulfilling each of the following after the 6 -month remission induction stage: (1) $\geq 50 \%$ decline in the IgG4-RD responder index (RI); (2) GC tapered to maintenance dose; and (3) no relapse during GC tapering. The patients not achieving the therapeutic goals were considered to have failed of remission induction.

Results: There were 26 patients in our cohort who failed of remission induction, including 16 (20.8\%) on GC monotherapy, and 10 (7.2\%) on combination therapy comprising GC and IM. The lacrimal gland and lung were most common sites of remission induction failure. Among the patients who relapsed during remission induction stage, 52.9\% had secondary relapse during follow-up. Eosinophilia, higher baseline Rl, more than five organs involved and dacryoadenitis were risk factors for remission induction failure with GC monotherapy, and the incidence of remission induction failure was $71.4 \%$ in the patients with more than three risk factors. After 6 -month treatment, the patients who failed of remission induction had significantly higher erythrocyte sedimentation rate (ESR), C-reactive protein (CRP) and lgG4.
\end{abstract}

Conclusion: In our cohort, 20.8\% of patients failed of remission induction with GC monotherapy, while $7.2 \%$ of patients failed of remission induction with combination therapy comprising GC and IM.

Keywords: IgG4-related disease, Remission, Relapse, Glucocorticoids, Immunosuppressive agents

\footnotetext{
* Correspondence: zhangwen91@sina.com

${ }^{\dagger}$ Equal contributors

'Department of Rheumatology, Peking Union Medical College Hospital (West

Campus), Chinese Academy of Medical Science \& Peking Union Medical

College, Key Laboratory of Rheumatology and Clinical Immunology, Ministry

of Education, No.41 Da Mu Cang, Western District, Beijing 100032, People's

Republic of China

Full list of author information is available at the end of the article
} 


\section{Background}

IgG4-related disease (IgG4-RD) is an immune-mediated systemic fibro-inflammatory disease, which is characterized by enlargement of the involved organs, elevated serum concentrations of IgG4, and pathology findings of dense lymphoplasmacytic infiltration enriched in IgG4positive plasma cells, storiform fibrosis, obliterative phlebitis and mild-to-moderate eosinophilia [1-4].

Glucocorticoid (GC) is the standard first-line agent for remission induction [5]. Patients with IgG4-RD typically respond well to initial GC treatment, with improvement of symptoms and signs, resolution of enlarged organs and decrease in serum IgG4 levels [6-10]. In addition, combination therapy comprising GC and immunosuppressive agents (IM) has also been shown effective, while the necessity of combination therapy from the beginning of treatment remains controversial [11-16].

Of note, there is a small minority of patients with less favorable response to remission induction, including those who have persistently active disease despite treatment, relapse during GC tapering or fail of GC tapering because of unstable disease [12, 17-20]. However, there are limited data on patients with less favorable response.

Here, we assessed the outcomes of remission induction in 215 patients with IgG4-RD in our prospective cohort, who were treated with GC alone or in combination with IM, and investigated the characteristics, prognosis, and risk factors among patients who failed of remission induction.

\section{Methods}

\section{Patient enrollment}

In our prospective cohort of patients with IgG4-RD in the Peking Union Medical college hospital [9], registered on ClinicalTrials.gov (ID: NCT01670695), 444 patients were enrolled from 2012 to 2017. Among them, we included all the newly diagnosed patients with IgG4-RD, who were initially treated with GC alone or in combination with IM, and had at least 6 months of follow up $(n=215)$. All patients satisfied the 2011 comprehensive diagnostic criteria for definite, probable or possible IgG4RD [21]. Patients with other rheumatic diseases, infectious diseases or malignancies were excluded. In addition, patients with conditions that could mimic IgG4-RD were excluded. The study protocol was approved by the Ethics Committee of Peking Union Medical College Hospital. All enrolled patients consented to attend this cohort study and signed written informed consent.

\section{Laboratory tests, imaging studies and histological examination}

Complete blood count, urinalysis, liver and renal function tests, erythrocyte sedimentation rate (ESR), hyper-sensitivity C-reactive protein (CRP), serum immunoglobulin levels and
IgG subclasses were tested. All patients underwent imaging examinations, including ultrasonography, computed tomography $(\mathrm{CT})$, magnetic resonance imaging (MRI) or positron emission tomography/computed tomography (PET-CT). Tissue biopsies were obtained from 112 patients, and samples were analyzed using previously described pathology methods [22].

\section{Treatment regimens}

All the patients were treated with prednisone at $0.5 \sim$ $1.0 \mathrm{mg} / \mathrm{kg}$ body weight/day $(30 \sim 60 \mathrm{mg} /$ day $)$. The initial dose of prednisone was continued for 2 weeks to 1 month, then gradually tapered by $5 \mathrm{mg}$ per 2 weeks to the maintenance dose of $5 \sim 10 \mathrm{mg} /$ day. Patients treated with additional IM $(\mathrm{n}=138)$ received cyclophosphamide (CTX) ( $\mathrm{n}=67)$, mycophenolate mofetil (MMF) $(\mathrm{n}=47)$, methotrexate (MTX) $(n=12)$, azathioprine (AZA) $(n=6)$, tripterysium glycosides (T2) $(\mathrm{n}=3)$, leflunomide (LEF) $(\mathrm{n}=1)$, cyclosporine A (CyA) $(\mathrm{n}=1)$ and CTX + $\operatorname{LEF}(\mathrm{n}=1)$.

\section{Assessment of disease activity and definition of relapse} Organ involvement was evaluated by medical history, physical examination, laboratory tests, imaging studies and tissue biopsies. Disease activity was assessed by the IgG4-RD responder index (RI) [23]. Follow-up visits were scheduled at months 1, 3 and 6, and once every $3 \sim 6$ months afterwards. During follow up, laboratory tests and imaging studies were performed when necessary. The patients were considered as having a relapse when they had recurrence of symptoms and signs or worsening on imaging studies, with or without re-elevation of serum IgG4.

\section{Therapeutic goals of remission induction and assessment of treatment outcomes}

The initial 6 months was defined as the remissioninduction stage, and the therapeutic goals of remission induction were defined as fulfilling each of the following after 6-month treatment: $(1) \geq 50 \%$ decline in the IgG4RD RI; (2) GC tapered to maintenance dose (prednisone $\leq 10 \mathrm{mg} /$ day); and (3) no relapse during GC tapering (within 6 months). The patients who reached the therapeutic goals were considered to have successful remission induction, while remission induction was considered to have failed in the other patients. In addition, the patients were grouped according to the treatment regimens they received during the remissioninduction stage. The flow scheme of patient enrollment, grouping and assessment of treatment outcomes is shown in Fig. 1.

\section{Statistical analysis}

Statistical analysis was performed using IBM SPSS (version 23) and R studio (version 3.4.0). Analysis by the 


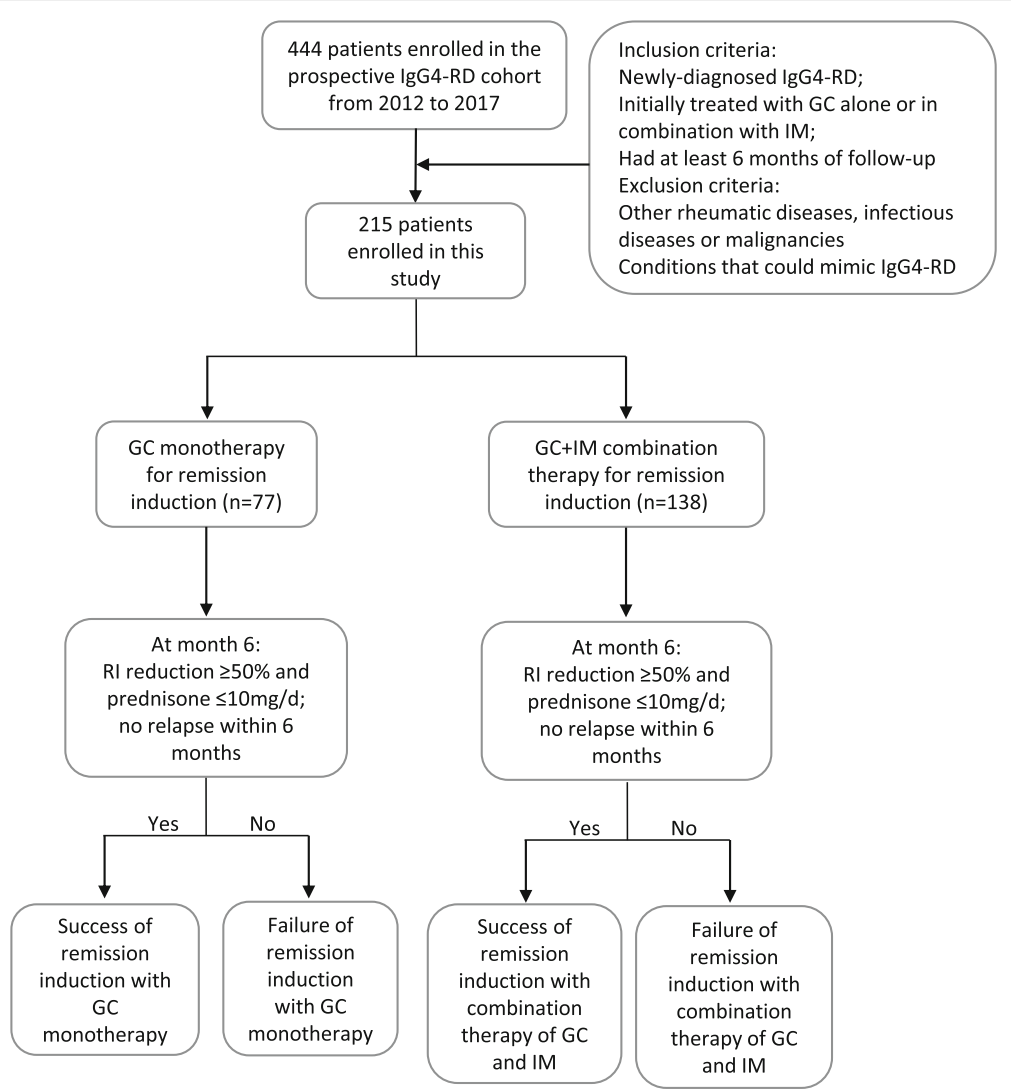

Fig. 1 Flow scheme of patient enrollment, grouping according to treatment and assessment of treatment outcomes. IgG4-RD, IgG4-related disease; GC, glucocorticoid; IM, immunosuppressive agents

Kolmogorov-Smirnov test revealed that data on age, disease duration, RI, number of organs involved, eosinophils, ESR, CRP, IgG, IgG4, IgG4/IgG and IgE were not normally distributed. All the continuous non-normally distributed data were presented as median (first quartile, third quartile) and analyzed by non-parametric test. Categorical variables were assessed by the chi-square test or Fisher's exact test. Univariate logistic regression was performed to investigate the risk factors for remission induction failure. Multivariate logistic regression was limited by the number of outcome events and the co-linearity among candidate risk factors. A $p$ value $<0$. 05 was considered statistically significant.

\section{Results}

\section{Patient demographic and baseline features}

Demographic and baseline features of 215 patients with IgG4-RD enrolled in the study are presented in Table 1. There were 148 male and 67 female patients (male to female 2.2:1). Median age was 54 years (range $9 \sim 83$ years). Median disease duration at the time of diagnosis was 12 months (range 10 days $~ 20$ years). The most common clinical manifestations included Mikulicz's disease, autoimmune pancreatitis, sclerosing cholangitis, retroperitoneal fibrosis, lung disease, sinusitis and lymphadenopathy.

\section{Response to treatment}

The median time of follow up was 22 months (range 6 60 months). The disease activity and GC dose at each visit are presented in Fig. 2. As shown in Fig. 2a and b, IgG4RD RI decreased over time, especially during the first 3 months of treatment. In response to remission induction therapy, $189(87.9 \%)$ patients reached remission without relapse, while $23(10.7 \%)$ patients relapsed during GC tapering and $3(1.4 \%)$ patients had persistently active disease despite 6-month treatment.

\section{Patients who failed of remission induction}

As shown in Table 2, according to our definition, there were $26(12.1 \%)$ patients in total who failed of remission induction, including 3 patients with decline in the IgG4-RD RI $<50 \%, 23$ patients who relapsed within 6 months and 17 patients who failed to have GC tapered to maintenance dose after 6 months. There was overlap between the patients who failed to have tapering of GC and patients with decline in the IgG4-RD RI $<50 \%$ or those who relapsed within 6 months. Significantly more 
Table 1 Baseline characteristics of patients with lgG4-RD $(n=215)$

\begin{tabular}{|c|c|}
\hline Variable & Value \\
\hline Sex (male:female) & $2.2: 1$ \\
\hline Age (years) & $54(46,62)$ \\
\hline \multicolumn{2}{|l|}{ Diagnosis, $n$ (\%) } \\
\hline Definite & $102(47.4 \%)$ \\
\hline Probable & $10(4.7 \%)$ \\
\hline Possible & $103(47.9 \%)$ \\
\hline Disease duration (months) & $12(4,36)$ \\
\hline IgG4-RD RI & $14(10,18)$ \\
\hline Allergy history, $n$ (\%) & $115(53.5 \%)$ \\
\hline \multicolumn{2}{|l|}{ Number of organs involved, $n(\%)$} \\
\hline $1 \sim 2$ & $78(36.3 \%)$ \\
\hline $3 \sim 4$ & $101(47.0 \%)$ \\
\hline$\geq 5$ & $36(16.7 \%)$ \\
\hline \multicolumn{2}{|l|}{ Organ involvement, $n$ (\%) } \\
\hline Mikulicz's disease & $138(64.2 \%)$ \\
\hline Dacryoadenitis & $95(44.2 \%)$ \\
\hline Sialoadenitis & $124(57.7 \%)$ \\
\hline Autoimmune pancreatitis & $91(42.3 \%)$ \\
\hline Sclerosing cholangitis & $58(27.0 \%)$ \\
\hline Retroperitoneal fibrosis & $50(23.3 \%)$ \\
\hline Lung disease & $62(28.8 \%)$ \\
\hline Kidney involvement & 19 (8.8\%) \\
\hline Lymphadenopathy & $139(64.7 \%)$ \\
\hline Sinusitis & $62(28.8 \%)$ \\
\hline Prostatitis & $30(14.0 \%)$ \\
\hline Skin involvement & $12(5.6 \%)$ \\
\hline Aortitis/periaortitis & $12(5.6 \%)$ \\
\hline Ophthalmic disease (except for dacryoadenitis) & $7(3.3 \%)$ \\
\hline Mediastinal fibrosis & $9(4.2 \%)$ \\
\hline Hepatopathy & $6(2.8 \%)$ \\
\hline Thyroiditis & $2(0.9 \%)$ \\
\hline Central nervous system involvement & $2(0.9 \%)$ \\
\hline \multicolumn{2}{|l|}{ Laboratory tests at baseline } \\
\hline Eosinophils (\%) & $3.2(1.6,6.3)$ \\
\hline $\operatorname{ESR}(\mathrm{mm} / \mathrm{h})$ & $25(10,59.5)$ \\
\hline $\mathrm{CRP}(\mathrm{mg} / \mathrm{L})$ & $3.78(1.15,10.81)$ \\
\hline $\operatorname{lgG}(\mathrm{g} / \mathrm{L})$ & $18.87(15.13,24.53)$ \\
\hline $\operatorname{lgG} 4$ (mg/L) & $8960(3500,18,600)$ \\
\hline $\lg \mathrm{E}(\mathrm{kU} / \mathrm{L})$ & $362.5(132.5,774)$ \\
\hline
\end{tabular}

All the continuous non-normally distributed data are presented as median (first quartile, third quartile)

IgG4-RD IgG4-related disease, $R /$ responder index, ESR erythrocyte sedimentation rate, $C R P$ hyper-sensitivity C-reactive protein patients failed of remission induction with GC monotherapy than with combination therapy $(16 / 77,20.8 \%$ vs $10 / 138,7.2 \%, p=0.008)$. Considering the heterogeneity of diagnostic status, we grouped the patients according to definite, probable and possible IgG4-RD, and found that the treatment outcomes were comparable in patients with different diagnostic status (Additional file 1). On the other hand, there was no significant difference in treatment-related side effects in patients treated with GC monotherapy or GC + IM combination therapy (Additional file 2).

\section{The organs that failed of remission induction}

Distribution of the organs that failed of remission induction, namely the organs in which persistently active disease or relapse occurred, are presented in Fig. 3a. The lacrimal gland and lung were the most common sites of remission induction failure. In the patients treated with GC monotherapy ( $\mathrm{n}=77)$, the highest incidence of remission induction failure occurred in the lung, lacrimal gland and bile duct, accounting for 29.4\% (5/17), 25.7\% $(9 / 35)$ and $13.6 \%(3 / 22)$, while in the patients treated with combination therapy ( $\mathrm{n}=138$ ), the organs with the highest incidence of remission induction failure included the pancreas $(7.0 \%, 4 / 57)$ and lacrimal gland $(6.7 \%, 4 / 60)$ (Fig. 3b).

Clinical features, treatments and outcomes in the patients who failed of remission induction

The clinical features, treatments and outcomes in the patients with persistently active disease or who relapsed during GC tapering are summarized individually in Additional files 3 and 4. Among the 23 patients who relapsed during GC tapering, 6 patients (26.1\%) were treated with an increased dose of GC in combination with additional or alternative IM, 12 patients (52.2\%) were treated with additional or alternative IM, 2 patients (8.7\%) were treated with an increased dose of GC and 3 patients $(13.0 \%)$ were treated with methylprednisolone (MP) pulse therapy, with or without adjustment of IM. Follow-up data after re-treatment were available in 21 patients, among whom 17 patients (81.0\%) reached remission and 9 patients $(52.9 \%)$ had another relapse after remission.

\section{Risk factors for remission induction failure}

We performed logistic regression analysis to identify baseline risk factors for failure of remission induction (Table 3). Univariate logistic regression analysis revealed that in the patients treated with GC monotherapy, eosinophilia (OR = 7.27, 95\% CI 2.05-25.74), higher baseline RI (highest quartile, $\mathrm{OR}=8.44,95 \%$ CI $1.48-48.14$ ), more organs involved ( $\geq 5$ organs, OR $=9.67,95 \% \mathrm{CI} 1.74-53.84$ ) and dacryoadenitis $(\mathrm{OR}=3.39,95 \% \mathrm{CI} 1.05-10.99)$ were 


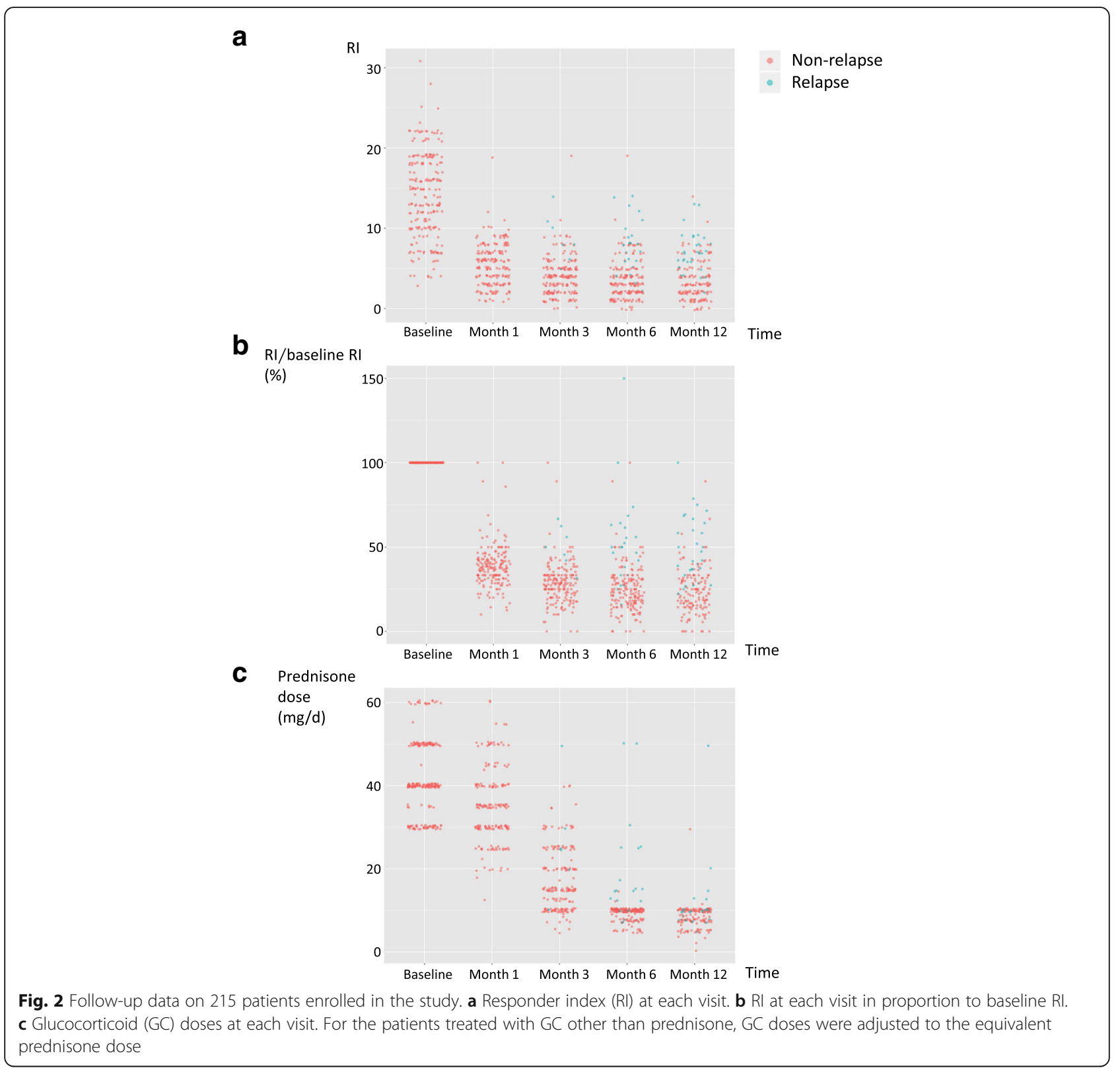

associated with increased risk of remission induction failure. In addition, higher baseline IgG4 could be a potential risk factor, although it was not statistically significant (IgG4 $>20 \times$ upper limit of normal (ULN), OR $=4.5,95 \%$ CI 0.89-22.67). In contrast, no significant association was identified between baseline characteristics and the risk of remission induction failure in the patients treated with combination therapy. Notably, the initial GC dose was not associated with the risk of remission induction failure in either group $(\mathrm{OR}=1.03,95 \% \mathrm{CI} 0.97-1.1$ and $\mathrm{OR}=0.97$, 95\% CI 0.9-1.05), neither was the duration of the initial dose (GC tapered before week 4 , OR $=0.75$, 95\% CI $0.2-$ 2.83 and $\mathrm{OR}=0.41,95 \% \mathrm{CI} 0.08-2.06)$. Multivariate logistic regression analysis was limited by the number of outcome events and the co-linearity among all the candidate risk factors.

To further investigate the impact of baseline risk factors on the incidence of remission induction failure, the patients treated with GC monotherapy were grouped according to the number of risk factors at baseline. As shown in Fig. 3c, the incidence of remission induction failure increased dramatically in the patients with more risk factors $(8 \%, 14.3 \%, 38.5 \%$ and $71.4 \%$ when having $0,1,2$ and 3-4 risk factors, respectively; $p=0.001$ when grouping the number of risk factors into categories of $0-1$ and $2-4$ ). 
Table 2 Baseline characteristics and outcomes of patients with lgG4-RD, grouped according to treatment

\begin{tabular}{|c|c|c|c|}
\hline & GC monotherapy $(n=77)$ & Combination therapy comprising GC and IM $(n=138)$ & $p$ value \\
\hline Sex (male:female) & $2.08: 1$ & $2.29: 1$ & 0.761 \\
\hline Age (years) & $54(45,61)$ & $54(48,62)$ & 0.723 \\
\hline Disease duration (months) & $12(4,24)$ & $12(4,36)$ & 0.649 \\
\hline IgG4-RD RI & $13(10,18)$ & $15(10,18)$ & 0.685 \\
\hline Allergy history & $42(54.5 \%)$ & $73(53.7 \%)$ & 1 \\
\hline Number of organs involved & & & 0.369 \\
\hline $1 \sim 2$ & $32(41.6 \%)$ & $46(33.3 \%)$ & \\
\hline $3 \sim 4$ & $35(45.5 \%)$ & $66(47.8 \%)$ & \\
\hline$\geq 5$ & $10(13.0 \%)$ & $26(18.8 \%)$ & \\
\hline \multicolumn{4}{|l|}{ Organ involvement } \\
\hline Mikulicz's disease & $51(66.2 \%)$ & $87(63.0 \%)$ & 0.659 \\
\hline Dacryoadenitis & 35 (45.5\%) & $60(43.5 \%)$ & 0.886 \\
\hline Sialoadenitis & $46(59.7 \%)$ & $78(56.5 \%)$ & 0.668 \\
\hline Autoimmune pancreatitis & $34(44.2 \%)$ & $57(41.3 \%)$ & 0.774 \\
\hline Sclerosing cholangitis & $22(28.6 \%)$ & $36(26.1 \%)$ & 0.749 \\
\hline Retroperitoneal fibrosis & $10(13.0 \%)$ & $40(29.0 \%)$ & 0.007 \\
\hline Lung disease & $17(22.1 \%)$ & $45(32.6 \%)$ & 0.118 \\
\hline Sinusitis & $25(32.5 \%)$ & $37(26.8 \%)$ & 0.433 \\
\hline Lymphadenopathy & $53(68.8 \%)$ & $86(62.3 \%)$ & 0.374 \\
\hline \multicolumn{4}{|l|}{ Laboratory tests at baseline } \\
\hline Eosinophils (\%) & $3.3(1.7,6.2)$ & $3.2(1.4,6.4)$ & 0.773 \\
\hline $\mathrm{ESR}(\mathrm{mm} / \mathrm{h})$ & $25(9,53)$ & $25(10,65)$ & 0.790 \\
\hline $\mathrm{CRP}(\mathrm{mg} / \mathrm{L})$ & $2.06(0.79,11.24)$ & $4.01(1.29,10.32)$ & 0.297 \\
\hline $\operatorname{lgG}(\mathrm{g} / \mathrm{L})$ & $18.83(14.24,25.54)$ & $18.9(15.2,23.68)$ & 0.747 \\
\hline $\operatorname{lgG} 4$ (mg/L) & $10,200(3590,18,800)$ & $8955(3480,18,075)$ & 0.554 \\
\hline $\lg E(k U / L)$ & $510(178,881)$ & $256(119,728)$ & 0.118 \\
\hline Initial GC dose & $40(35,50)$ & $40(40,50)$ & 0.567 \\
\hline \multicolumn{4}{|l|}{ Outcomes at month 6} \\
\hline RI reduction ${ }^{a}<50 \%$ & $2(2.6 \%)$ & $1(0.7 \%)$ & 0.292 \\
\hline Relapse & $14(18.2 \%)$ & $9(6.5 \%)$ & 0.011 \\
\hline Failure of GC tapering & $8(10.4 \%)$ & $9(6.5 \%)$ & 0.429 \\
\hline Failure of remission induction & $16(20.8 \%)$ & $10(7.2 \%)$ & 0.008 \\
\hline
\end{tabular}

All the continuous non-normally distributed data were presented as median (first quartile, third quartile)

IgG4-RD IgG4-related disease, RI responder index, ESR erythrocyte sedimentation rate, CRP hyper-sensitivity C-reactive protein, GC glucocorticoid, IM immunosuppressive agents

${ }^{a}$ Here we considered the maximum reduction during follow up, so that re-elevation due to relapse was not taken into account

\section{Comparison of serological features at month 6 in the patients who succeeded or failed of remission induction}

To further investigate the characteristics of the patients who failed of remission induction, we compared the serological features after the remission-induction stage (at month 6) in the patients with success or failure of remission induction (Fig. 4). Interestingly, the patients who failed of remission induction had significantly higher $\operatorname{ESR}(\mathrm{mm} / \mathrm{h})(11(4,27)$ vs $5(2,9), p=0.0056)$, CRP (mg/L) $(2.60(0.70,29.94)$ vs $0.73(0.36,1.83), p=0$.
0033) and IgG4 (mg/L) (3570 (2413, 6443) vs 1870 (859, 3928), $p=0.0047)$, whereas IgG was comparable between these two groups.

\section{Discussion}

In this article, we assessed the outcome of remission induction in 215 patients with IgG4-RD, who were treated with GC alone or in combination with IM, and focused on the subpopulation of patients with less favorable response to treatment. 

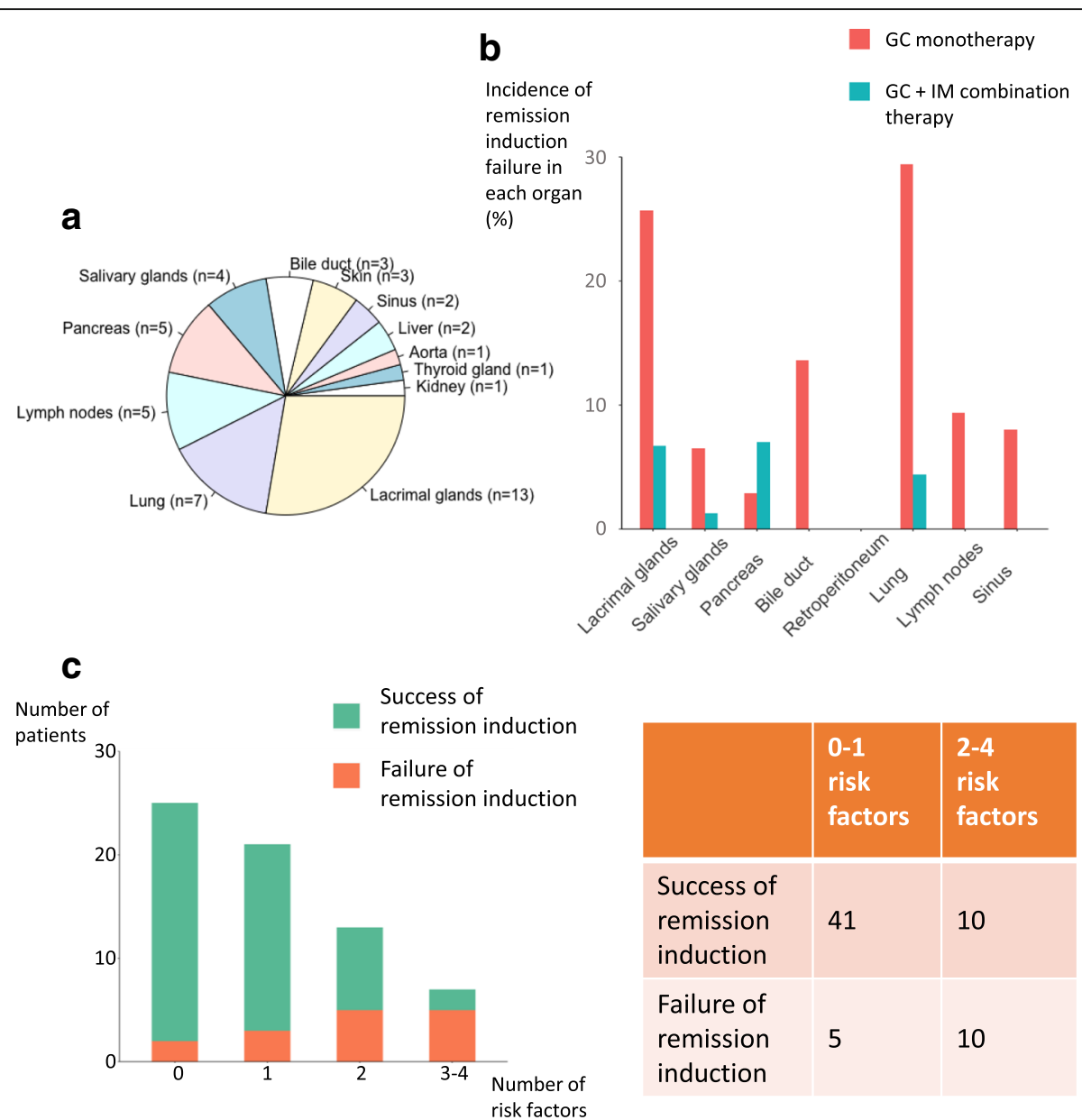

Fisher's exact test: $p=0.001$

Fig. 3 Organs that failed of remission induction. a Distribution of the organs that failed of remission induction. $\mathbf{b}$ Incidence of remission induction failure in each organ. The incidence was calculated as number of remission induction failures occurred in each organ/total number of patients with that particular organ involvement. c Treatment outcomes in the patients treated with glucocorticoid (GC) monotherapy, grouped according to the number of risk factors they had. Risk factors included eosinophilia, baseline responder index (RI) in the highest quartile, $\geq 5$ organs involved and dacryoadenitis. IM, immunosuppressive agents

Treat-to-target approaches, in which treatment regimens are escalated or adjusted until a specific target is reached, have been applied as routine management of patients with a variety of chronic diseases, and have been demonstrated to improve treatment outcomes significantly [24-28]. However, the therapeutic goals in IgG4-RD have not been clearly defined. It has been reported that in patients with IgG4-RD, persistently active disease could lead to fibrosis and irreversible organ damage $[1,2,29]$. Therefore, early intervention is critical, and it is also important to clarify the therapeutic goals of remission induction and confirm that the patients are receiving adequate treatment. Here, based on our experience, we defined the therapeutic goals of remission induction as fulfilling each of the following after 6-month treatment: $(1) \geq 50 \%$ decline in the IgG4-RD RI; (2) GC tapered to maintenance dose (prednisone $\leq 10 \mathrm{mg} /$ day); and (3) no relapse during GC tapering (within 6 months). The patients not fulfilling the treatment goals were considered as failure of remission induction. We are aware of the possibility that the serum IgG4 level might not be lowered even if the treatment was effective, resulting in unsatisfactory decline in the IgG4-RD RI, and therefore some patients even in clinical remission might be classified as remission induction failure. However, in our cohort, only three patients failed of remission induction based on this criterion, and all three presented with persistently active disease in the affected organs, accompanied by failure of GC tapering to maintenance dose. Further validation is required for the treatment goals proposed by us.

Most of the patients reached remission through 6-month treatment, while 26 patients (12.1\%) failed of remission induction (16 (20.8\%) with GC monotherapy, and 10 (7.2\%) with combination therapy comprising GC and IM). The baseline features were almost comparable between the two 
Table 3 Univariate logistic regression analysis of risk factors for remission induction failure

\begin{tabular}{|c|c|c|c|c|}
\hline & \multicolumn{2}{|l|}{ GC monotherapy } & \multicolumn{2}{|c|}{ GC + IM combination therapy } \\
\hline & OR $(95 \% \mathrm{Cl})$ & $p$ value & OR $(95 \% \mathrm{Cl})$ & $p$ value \\
\hline Male sex & $1.07(0.33,3.51)$ & 0.907 & $4.24(0.52,34.6)$ & 0.177 \\
\hline Age (continuous) & $0.97(0.93,1.01)$ & 0.103 & $1(0.95,1.05)$ & 0.901 \\
\hline Disease duration ( $\geq 12$ months) & $1.02(0.34,3.1)$ & 0.971 & $0.39(0.1,1.58)$ & 0.186 \\
\hline Allergy history & $1.51(0.49,4.68)$ & 0.474 & $2.12(0.52,8.58)$ & 0.291 \\
\hline Eosinophilia & $7.27(2.05,25.74)$ & 0.002 & $1.46(0.39,5.48)$ & 0.576 \\
\hline Elevation of ESR & $1.13(0.33,3.88)$ & 0.847 & $1.03(0.24,4.54)$ & 0.966 \\
\hline Elevation of CRP & $1.65(0.4,6.86)$ & 0.493 & $1.22(0.28,5.39)$ & 0.791 \\
\hline \multicolumn{5}{|l|}{$\lg G$} \\
\hline First quartile (median $12.78 \mathrm{~g} / \mathrm{L}$ ) & Ref & Ref & Ref & Ref \\
\hline Second quartile (median $17.25 \mathrm{~g} / \mathrm{L}$ ) & $0.50(0.08,3.15)$ & 0.461 & $0.94(0.12,7.08)$ & 0.949 \\
\hline Third quartile (median $21.30 \mathrm{~g} / \mathrm{L}$ ) & $1.13(0.21,6.14)$ & 0.892 & $1.76(0.3,10.31)$ & 0.532 \\
\hline Fourth quartile (median $31.67 \mathrm{~g} / \mathrm{L}$ ) & $1.41(0.33,5.98)$ & 0.644 & $1.12(0.15,8.49)$ & 0.916 \\
\hline \multicolumn{5}{|l|}{$\operatorname{lgG} 4$} \\
\hline $\lg G 4 \leq 3 \times \cup L N$ & Ref & Ref & Ref & Ref \\
\hline $3 \times \cup L N<\lg G 4 \leq 10 \times \cup L N$ & $1(0.2,5.04)$ & 1 & $\operatorname{Ref}^{a}$ & $\operatorname{Ref}^{a}$ \\
\hline $10 \times \mathrm{ULN}<\operatorname{lgG} 4 \leq 20 \times \mathrm{ULN}$ & $1.64(0.28,9.58)$ & 0.585 & $1.46(0.35,6.05)$ & 0.604 \\
\hline $\operatorname{lgG} 4>20 \times$ ULN & $4.5(0.89,22.67)$ & 0.068 & $N A^{b}$ & $N A^{b}$ \\
\hline \multicolumn{5}{|l|}{$\lg E$} \\
\hline First quartile (median 75.5 kU/L) & Ref & Ref & Ref & Ref \\
\hline Second quartile (median 203 kU/L) & $1.45(0.11,18.96)$ & 0.775 & $4.5(0.44,46.38)$ & 0.206 \\
\hline Third quartile (median 529 kU/L) & $1.07(0.08,13.65)$ & 0.96 & $1.67(0.1,28.32)$ & 0.724 \\
\hline Fourth quartile (median 1403 kU/L) & $3.64(0.35,37.46)$ & 0.278 & $3.16(0.27,37.27)$ & 0.361 \\
\hline \multicolumn{5}{|l|}{ Rl } \\
\hline First quartile (median 7) & Ref & Ref & Ref & Ref \\
\hline Second quartile (median 13) & $0.95(0.12,7.44)$ & 0.961 & $1.82(0.24,13.81)$ & 0.563 \\
\hline Third quartile (median 16) & $2.92(0.47,18.37)$ & 0.253 & $1.08(0.14,8.07)$ & 0.939 \\
\hline Fourth quartile (median 21) & $8.44(1.48,48.14)$ & 0.016 & $2.76(0.47,16.09)$ & 0.259 \\
\hline \multicolumn{5}{|l|}{ Number of organs involved } \\
\hline $1 \sim 2$ & Ref & Ref & Ref & Ref \\
\hline $3 \sim 4$ & $2.86(0.69,11.93)$ & 0.148 & $0.92(0.2,4.34)$ & 0.921 \\
\hline$\geq 5$ & $9.67(1.74,53.84)$ & 0.01 & $1.87(0.35,10.02)$ & 0.465 \\
\hline \multicolumn{5}{|l|}{ Organ involvement } \\
\hline Mikulicz's disease & $4.54(0.95,21.78)$ & 0.059 & $0.56(0.15,2.04)$ & 0.38 \\
\hline Dacryoadenitis & $3.39(1.05,10.99)$ & 0.042 & $1.33(0.37,4.81)$ & 0.667 \\
\hline Sialoadenitis & $1.63(0.51,5.28)$ & 0.412 & $0.75(0.21,2.73)$ & 0.667 \\
\hline Autoimmune pancreatitis & $1.35(0.45,4.06)$ & 0.598 & $0.94(0.25,3.51)$ & 0.931 \\
\hline Sclerosing cholangitis & $0.8(0.23,2.8)$ & 0.723 & $2(0.53,7.54)$ & 0.306 \\
\hline Retroperitoneal fibrosis & $N A^{b}$ & $N A^{b}$ & $0.25(0.03,2.07)$ & 0.2 \\
\hline Lung disease & $2.73(0.82,9.09)$ & 0.103 & $1.41(0.38,5.29)$ & 0.606 \\
\hline Initial GC dose & $1.03(0.97,1.1)$ & 0.271 & $0.97(0.9,1.05)$ & 0.441 \\
\hline GC tapered before week 4 & $0.75(0.2,2.83)$ & 0.671 & $0.41(0.08,2.06)$ & 0.279 \\
\hline
\end{tabular}

Abbreviations: GC glucocorticoid, IM immunosuppressive agents, $R I$ responder index, ESR erythrocyte sedimentation rate, CRP hyper-sensitivity C-reactive protein, OR odds ratio, Ref reference, ULN upper limit of normal, NA not applicable

${ }^{a}$ The first two groups were combined as $0 \%$ remission induction failure occurred in the patients with $\operatorname{lgG} 4 \leq 3 \times$ ULN

${ }^{\mathrm{b}}$ Not applicable as $0 \%$ remission induction failure occurred in certain groups 

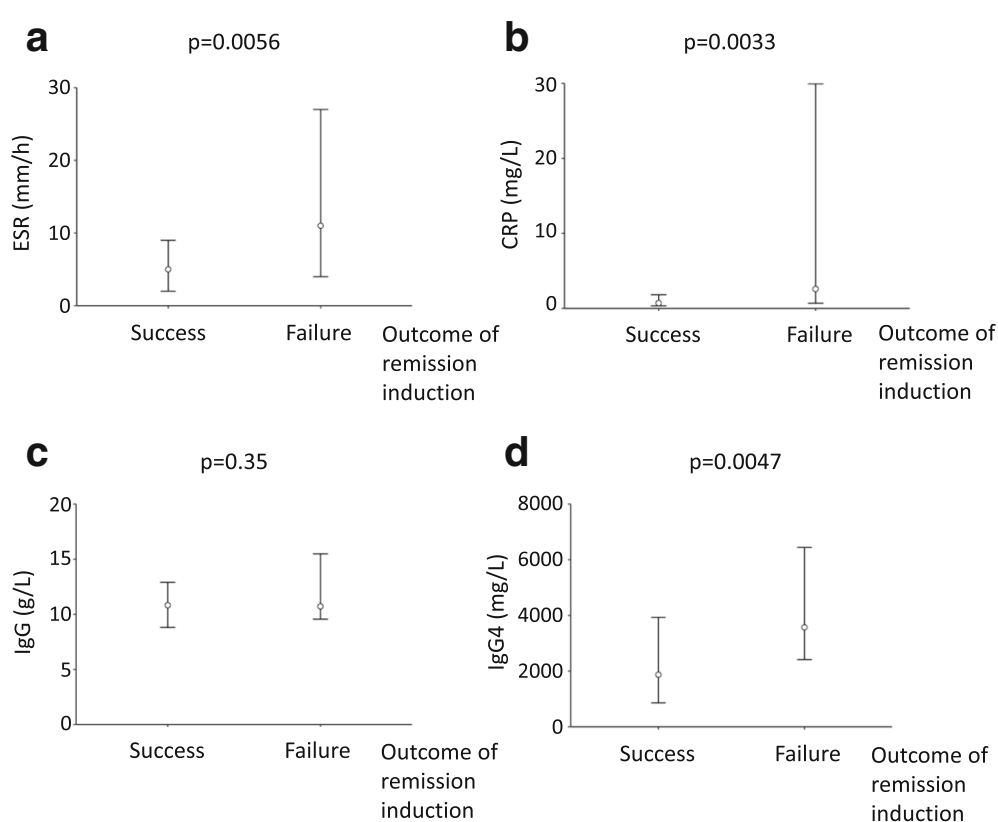

Fig. 4 Serological features at month 6 in patients who succeeded or failed of remission induction. Levels of serum erythrocyte sedimentation rate (ESR) (a), hyper-sensitivity C-reactive protein (CRP) (b), IgG (c) and IgG4 (d) at month 6 were compared between the patients who succeeded or failed of remission induction. Data represent the median (first quartile, third quartile) of each parameter

groups (Table 2), while the incidence of remission induction failure in the patients treated with GC monotherapy was significantly higher $(p=0.008)$. On the other hand, the patients treated with combination therapy did not experience more side effects compared to those treated with GC monotherapy (Additional file 2). It remains controversial whether some patients require the combination of GC and IM from the beginning of treatment [5]. Our data, along with previously published data showing that the patients treated with GC + CTX had a significantly higher rate of complete remission and lower rate of relapse [11], support the necessity of combination therapy with GC and IM as the initial treatment in certain patients.

Failure of remission induction most commonly occurred in the lacrimal glands and lungs (Fig. 3a). In the patients treated with GC monotherapy, lungs, lacrimal glands and bile duct had the highest incidence of remission induction failure $(29.4 \%, 25.7 \%$ and $13.6 \%$, respectively), indicating that the patients with involvement of these organs need more intensive follow up (Fig. 3b). In the patients treated with a combination of GC and IM, the overall incidence of remission induction failure was low, with relatively higher incidence in the pancreas and lacrimal glands (Fig. 3b).

Among the 23 patients who relapsed during GC tapering, re-treatment either with more intensive GC therapy or with the addition of or change in IM was effective in most of the patients $(17 / 21,81.0 \%)$, which is consistent with data from other studies $[6,12,30]$. Of note, among the patients who relapsed, secondary relapse occurred in $52.9 \%$, which is much higher than the overall relapse rate with initial therapy $(21.4 \%)$, indicating that the patients who have relapsed are susceptible to another relapse, and they need more intensive follow up.

Of note, there are four patients in our cohort (patient number 3, 15, 16 and 24) (Additional files 3 and 4), who had persistently active disease refractory to multiple treatment regimens. Although unresponsiveness to various treatment regimens is rare in IgG4-RD, diagnosis of IgG4-RD in those patients was confirmed by biopsy, and conditions that could mimic IgG4-RD, including antineutrophil-cytoplasmic antibody (ANCA)-associated vasculitis, multicentric Castleman's disease, Kimura disease, Rosai-Dorfman disease and cancer, etc., were excluded. In accordance with our data, Wallace et al. reported three patients with IgG4-RD unresponsive to treatment with GC and/or rituximab [18]. Sun et al. reported three cases of IgG4-related lung disease, that were either unresponsive to a routine dose of GC, or failed GC tapering because of recurrent disease [20]. Taken together, it is possible that a small minority of patients with IgG4-RD is refractory to treatment, and further study on the management of those patients is required.

In the patients treated with GC monotherapy, eosinophilia, higher baseline RI, more than five organs involved and dacryoadenitis were identified as risk factors for remission induction failure (Table 3). Consistent with our data, it has been reported that higher baseline RI and 
greater number of organs involved are associated with worse outcomes with GC therapy [11, 19]. Eosinophilia is a common clinical manifestation in patients with IgG4RD [3, 4]. Interestingly, it has been proposed that eosinophilia is associated with the inherent characteristics of IgG4-RD, rather than underlying allergy [31]. Our previous study also demonstrated that peripheral eosinophils was associated with disease severity, indicated by the number of organs involved and serum IgG4 levels [32]. Here, we also found that eosinophilia, rather than allergy history, is associated with treatment outcome. The etiology of eosinophilia in patients with IgG4-RD and its role in pathogenesis requires further investigation. As for particular organ involvement, dacryoadenitis is a newly discovered risk factor for worse outcome. In contrast to our study, it has been reported that in the patients with autoimmune pancreatitis, the presence of sclerosing cholangitis is a risk factor for relapse [6]. The discrepancy could be caused by the difference in disease subtypes and observation endpoints (failure of remission induction vs relapse).

When the patients treated with GC monotherapy were grouped according to the number of risk factors at baseline, we identified a trend towards higher incidence of remission induction failure in the patients with more risk factors (Fig. 3c). Of note, in the patients with more than three risk factors, the incidence of remission induction failure was as high as $71.4 \%$. As aforementioned, remission induction failure was more common in the patients treated with GC monotherapy. Taken together, our data suggest the necessity of combination therapy with GC and IM for the patients with more than three risk factors.

In addition, after the remission-induction stage, serum ESR, CRP and IgG4 was significantly higher in the patients who failed of remission induction (Fig. 4), indicating the usefulness of these serological parameters to assess response to treatment. Consistent with our data, it has been reported that in patients with autoimmune pancreatitis, persistent elevation of IgG4 level after treatment is associated with persistent abnormalities of the pancreas and bile duct and more frequent relapse [7]. It has been proposed that the quantitation of circulating plasmablasts is better for monitoring disease activity than IgG4 level $[18,33]$. However, considering the clinical availability, it is still valid to monitor serum IgG4 during follow up.

Our study also has some limitations. The biopsy-proven diagnosis rate was $52.1 \%$, and the remaining patients were diagnosed based on clinical manifestation and serum IgG4 levels. However, all the patients without biopsy-proven diagnosis presented with typical features of IgG4-RD, conditions that could mimic IgG4-RD were excluded and the treatment outcomes were comparable in the patients with different diagnostic status (Additional file 1). In addition, the therapy regimens are varied between patients, including the initial doses of $\mathrm{GC}$, the duration of initial dose and the type of IM. However, in logistic regression analysis there was no association between the risk of remission induction failure and either the initial GC dose or duration of the initial dose (Table 3). In addition, previous research from our group found no significant difference in the efficacy of high and medium doses of GC in the induction of remission [34]. We infer that the difference in GC dose and tapering did not cause much bias. On the other hand, the differences in usage of IM might be the reason why no risk factors for remission induction failure were identified in the combination therapy group.

\section{Conclusions}

In conclusion, according to our definition, 26 patients (12.1\%) failed of remission induction (16 (20.8\%) with GC monotherapy, and 10 (7.2\%) with combination therapy comprising GC and IM). The lacrimal glands and lungs were the most common sites of remission induction failure. Eosinophilia, higher baseline RI, involvement of more than five organs and dacryoadenitis were risk factors for remission induction failure with GC monotherapy, and combination therapy of GC and IM should be considered for patients with more than three of these risk factors. After the remission-induction stage, patients who failed of remission induction had significantly higher ESR, CRP and IgG4.

\section{Additional files}

Additional file 1: Outcomes of remission induction in the patients diagnosed with definite, probable and possible lgG4-RD. (DOCX $71 \mathrm{~kb}$ )

Additional file 2: Side effects observed during treatment. (DOCX $68 \mathrm{~kb}$ ) Additional file 3: Clinical features, treatments and outcomes of the patients with persistently active disease. (DOCX $70 \mathrm{~kb}$ )

Additional file 4: Clinical features, treatments and outcomes of the patients who relapsed during GC tapering. (DOCX $130 \mathrm{~kb}$ )

\section{Abbreviations \\ AZA: Azathioprine; CRP: Hyper-sensitivity C-reactive protein; CT: Computed tomography; CTX: Cyclophosphamide; CyA: Cyclosporine A; ESR: Erythrocyte sedimentation rate; GC: Glucocorticoids; IgG4-RD: IgG4-related disease; IM: Immunosuppressive agents; LEF: Leflunomide; MMF: Mycopheolatemofetil; MP: Methylprednisolone; MRI: Magnetic resonance imaging; MTX: Methotrexate; OR: Odds ratio; PET-CT: Positron emission tomography/computed tomography; Ref: Reference; RI: Responder index; T2: Tripterysium glycosides; ULN: Upper limit of normal}

\section{Acknowledgements}

Not applicable.

\section{Funding}

This work was supported by the National Natural Science Foundation of China (81373190 and 81571587), Science Foundation of Beijing (7172178), CAMS Initiative for Innovative Medicine (2017-12M-3-001), and The National Key Research and Development Program of China (2016YFC0901500). 


\section{Availability of data and materials}

The datasets used and/or analyzed during the current study are available from the corresponding author on reasonable request.

\section{Authors' contributions}

LW designed the research, analyzed data and wrote the manuscript. PZ collected data, analyzed data and revised the manuscript. RF helped with pathology diagnosis. MW, YL, LP and YF participated in case and data collection. $X Z, Y Z$ and $X Z$ helped optimize the research and proofread the paper. WZ and FZ designed the study and wrote the manuscript. All authors read and approved the final manuscript.

\section{Ethics approval and consent to participate}

The study protocol was approved by the Ethics Committee of Peking Union Medical College Hospital. All enrolled patients consented to attend this cohort study and gave written, signed informed consent.

\section{Consent for publication}

We obtained consent for publication from all the individuals on whom detailed information was included in the manuscript.

\section{Competing interests}

The authors declare that they have no competing interests.

\section{Publisher's Note}

Springer Nature remains neutral with regard to jurisdictional claims in published maps and institutional affiliations.

\section{Author details}

'Department of Rheumatology, Peking Union Medical College Hospital (West Campus), Chinese Academy of Medical Science \& Peking Union Medical College, Key Laboratory of Rheumatology and Clinical Immunology, Ministry of Education, No.41 Da Mu Cang, Western District, Beijing 100032, People's Republic of China. ${ }^{2} T$ singhua University School of Medicine, Beijing 100086, China. ${ }^{3}$ Department of Stomatology, Peking Union Medical College Hospital, Beijing, China. ${ }^{4}$ Department of Pathology, Peking Union Medical College Hospital, Beijing, China. ${ }^{5}$ Department of Gastroenterology, Peking Union Medical College Hospital, Beijing, China.

Received: 11 January 2018 Accepted: 14 March 2018

Published online: 10 April 2018

\section{References}

1. Stone JH, Zen Y, Deshpande V. IgG4-related disease. N Engl J Med. 2012;366(6):539-51.

2. Kamisawa T, Zen $Y$, Pillai $\mathrm{S}$, Stone JH. IgG4-related disease. Lancet (London, England). 2015;385(9976):1460-71.

3. Stone $J H$, Brito-Zeron P, Bosch X, Ramos-Casals M. Diagnostic approach to the complexity of IgG4-related disease. Mayo Clin Proc. 2015;90(7):927-39.

4. Brito-Zeron P, Bosch X, Ramos-Casals M, Stone JH. IgG4-related disease: advances in the diagnosis and treatment. Best Pract Res Clin Rheumatol. 2016;30(2):261-78.

5. Khosroshahi A, Wallace ZS, Crowe JL, Akamizu T, Azumi A, Carruthers MN, et al. International consensus guidance statement on the management and treatment of IgG4-related disease. Arthritis Rheumatol. 2015;67(7):1688-99.

6. Hart PA, Kamisawa T, Brugge WR, Chung JB, Culver EL, Czako L, et al. Long-term outcomes of autoimmune pancreatitis: a multicentre, international analysis. Gut. 2013;62(12):1771-6.

7. Kamisawa T, Shimosegawa T, Okazaki K, Nishino T, Watanabe H, Kanno A et al. Standard steroid treatment for autoimmune pancreatitis. Gut. 2009;58(11):1504-7.

8. Sandanayake NS, Church NI, Chapman MH, Johnson GJ, Dhar DK, Amin Z, et al. Presentation and management of post-treatment relapse in autoimmune pancreatitis/immunoglobulin G4-associated cholangitis. Clin Gastroenterol Hepatol. 2009;7(10):1089-96.

9. Lin W, Lu S, Chen H, Wu Q, Fei Y, Li M, et al. Clinical characteristics of immunoglobulin G4-related disease: a prospective study of 118 Chinese patients. Rheumatology (Oxford). 2015:54(11):1982-90.

10. Campochiaro C, Ramirez GA, Bozzolo EP, Lanzillotta M, Berti A, Baldissera E, et al. IgG4-related disease in Italy: clinical features and outcomes of a large cohort of patients. Scand J Rheumatol. 2016;45(2):135-45.
11. Yunyun F, Yu C, Panpan Z, Hua C, Di W, Lidan Z, et al. Efficacy of Cyclophosphamide treatment for immunoglobulin G4-related disease with addition of glucocorticoids. Sci Rep. 2017;7(1):6195.

12. Hart PA, Topazian MD, Witzig TE, Clain JE, Gleeson FC, Klebig RR, et al. Treatment of relapsing autoimmune pancreatitis with immunomodulators and rituximab: the Mayo Clinic experience. Gut. 2013;62(11):1607-15.

13. Bosco JJ, Suan D, Varikatt W, Lin MW. Extra-pancreatic manifestations of IgG4-related systemic disease: a single-centre experience of treatment with combined immunosuppression. Intern Med J. 2013;43(4):417-23.

14. Della-Torre E, Campochiaro C, Bozzolo EP, Dagna L, Scotti R, Nicoletti R, et al. Methotrexate for maintenance of remission in lgG4-related disease. Rheumatology (Oxford). 2015:54(10):1934-6.

15. Wang Y, Li K, Gao D, Luo G, Zhao Y, Wang X, et al. Combination therapy of leflunomide and glucocorticoids for the maintenance of remission in patients with lgG4-related disease: a retrospective study and literature review. Intern Med J. 2017;47(6):680-9.

16. Gupta N, Mathew J, Mohan H, Chowdhury SD, Kurien RT, Christopher DJ, et al. Addition of second-line steroid sparing immunosuppressants like mycophenolate mofetil improves outcome of immunoglobulin G4-related disease (IgG4-RD): a series from a tertiary care teaching hospital in South India. Rheumatol Int. 2018:38(2):203-9.

17. McMahon BA, Novick T, Scheel PJ, Bagnasco S, Atta MG. Rituximab for the treatment of lgG4-related tubulointerstitial nephritis: case report and review of the literature. Medicine (Baltimore). 2015;94(32):e1366.

18. Wallace ZS, Mattoo H, Mahajan VS, Kulikova M, Lu L, Deshpande V, et al. Predictors of disease relapse in IgG4-related disease following rituximab. Rheumatology (Oxford, England). 2016;55(6):1000-8.

19. Liu W, Chen W, He X, Qu Q, Hong T, Li B. Poor response of initial steroid therapy for lgG4-related sclerosing cholangitis with multiple organs affected. Medicine (Baltimore). 2017:96(12):e6400.

20. Sun X, Peng M, Hou X, Feng R, Xu Z. Refractory lgG4-related lung disease with constitutional symptoms and severe inflammation. Am J Respir Crit Care Med. 2014;189(3):374-5

21. Umehara H, Okazaki K, Masaki Y, Kawano M, Yamamoto M, Saeki T, et al. Comprehensive diagnostic criteria for lgG4-related disease (lgG4-RD), 2011 Mod Rheumatol. 2012;22(1):21-30.

22. Chen $H$, Lin W, Wang $Q$, Wu Q, Wang L, Fei $Y$, et al. IgG4-related disease in a Chinese cohort: a prospective study. Scand J Rheumatol. 2014;43(1):70-4.

23. Carruthers MN, Stone JH, Deshpande V, Khosroshahi A. Development of an IgG4-RD responder index. Int J Rheumatol. 2012;2012:259408.

24. Franklyn K, Hoi A, Nikpour M, Morand EF. The need to define treatment goals for systemic lupus erythematosus. Nat Rev Rheumatol. 2014;10(9):567-71.

25. Smolen JS, Breedveld FC, Burmester GR, Bykerk V, Dougados M, Emery P, et al. Treating rheumatoid arthritis to target: 2014 update of the recommendations of an international task force. Ann Rheum Dis. 2016;75(1):3-15.

26. Verdecchia P, Staessen JA, Angeli F, de Simone G, Achilli A, Ganau A, et al. Usual versus tight control of systolic blood pressure in non-diabetic patients with hypertension (Cardio-Sis): an open-label randomised trial. Lancet (London, England). 2009;374(9689):525-33.

27. Verstappen SM, Jacobs JW, van der Veen MJ, Heurkens AH, Schenk Y, ter Borg EJ, et al. Intensive treatment with methotrexate in early rheumatoid arthritis: aiming for remission. Computer Assisted Management in Early Rheumatoid Arthritis (CAMERA, an open-label strategy trial). Ann Rheum Dis 2007:66(11):1443-9.

28. Grigor C, Capell H, Stirling A, McMahon AD, Lock P, Vallance R, et al. Effect of a treatment strategy of tight control for rheumatoid arthritis (the TICORA study): a single-blind randomised controlled trial. Lancet (London, England). 2004;364(9430):263-9.

29. Shimizu Y, Yamamoto M, Naishiro Y, Sudoh G, Ishigami K, Yajima H, et al. Necessity of early intervention for lgG4-related disease-delayed treatment induces fibrosis progression. Rheumatology (Oxford). 2013;52(4):679-83.

30. Brito-Zeron P, Kostov B, Bosch X, Acar-Denizli N, Ramos-Casals M, Stone JH. Therapeutic approach to lgG4-related disease: a systematic review. Medicine (Baltimore). 2016;95(26):e4002.

31. Della Torre E, Mattoo H, Mahajan VS, Carruthers M, Pillai S, Stone JH. Prevalence of atopy, eosinophilia, and IgE elevation in IgG4-related disease. Allergy. 2014;69(2):269-72.

32. Chen $Y$, Zhao JZ, Feng RE, Shi JH, Li XM, Fei YY, et al. Types of organ involvement in patients with immunoglobulin G4-related disease. Chin Med J. 2016;129(13):1525-32. 
33. Wallace ZS, Mattoo H, Carruthers M, Mahajan VS, Della Torre E, Lee H, et al. Plasmablasts as a biomarker for lgG4-related disease, independent of serum IgG4 concentrations. Ann Rheum Dis. 2015;74(1):190-5.

34. Wu Q, Chang J, Chen H, Chen Y, Yang H, Fei Y, et al. Efficacy between high and medium doses of glucocorticoid therapy in remission induction of IgG4-related diseases: a preliminary randomized controlled trial. Int J Rheum Dis. 2017;20(5):639-46.

Submit your next manuscript to BioMed Central and we will help you at every step:

- We accept pre-submission inquiries

- Our selector tool helps you to find the most relevant journal

- We provide round the clock customer support

- Convenient online submission

- Thorough peer review

- Inclusion in PubMed and all major indexing services

- Maximum visibility for your research

Submit your manuscript at www.biomedcentral.com/submit 